a)

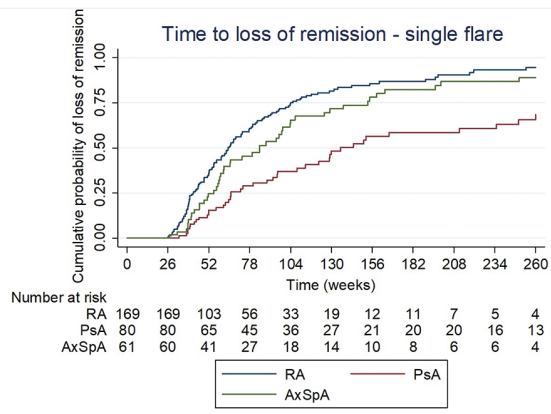

b)

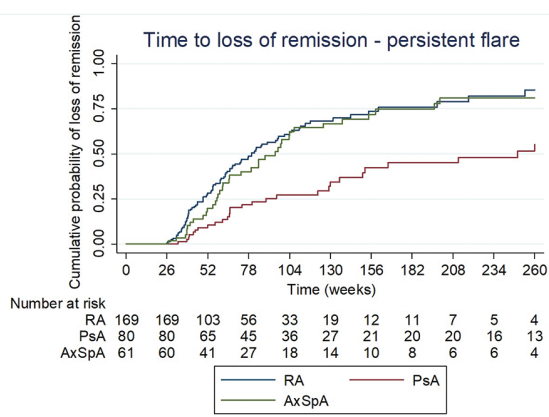

Figure 3. - time to loss of remission considering a single flare (a) vs. persistent flare (b) by diagnosis, all patients (minimum 150 days in remission).

Conclusion: 6 vs. 12 months of sustained remission did not influence the subsequent rate of LOR. Smoking, the type of TNFi, female gender and duration of disease until biologic treatment were identified as predictors of LOR.

Disclosure of Interests: Pedro Ávila-Ribeiro: None declared, Joao Eurico Fonseca: None declared, Elsa Vieira-Sousa Grant/research support from: MSD, Novartis

DOI: 10.1136/annrheumdis-2019-eular.8034

\section{FRI0660 LONG- AND SHORT-TERM ASSOCIATION OF LOW- GRADE SYSTEMIC INFLAMMATION WITH CARDIOVASCULAR MORTALITY}

Jan Leipe $^{1}$, Anna-Isabelle Kaelsch ${ }^{2}$, Marcus Kleber ${ }^{2}$, Hubert Scharnagl ${ }^{3}$, Wolfgang Sattler ${ }^{3}$, Winfried März ${ }^{4}$, Ernst Malle ${ }^{3}$, Bernhard. Kraemer ${ }^{2} .{ }^{1}$ University Medical Centre Mannheim, Division of Rheumatology, Mannheim, Germany; ${ }^{2}$ University Medical Centre Mannheim, Department of Medicine V, Mannheim, Germany; ${ }^{3}$ Medical University of Graz, Graz, Austria; ${ }^{4}$ Synlab Academy, Mannheim, Germany

Background: Evidence suggests a critical role for inflammation in the pathogenesis of coronary artery/heart disease (CAD/CHD), implicating inflammatory molecules as central mediators of chronic inflammatory processes within the vascular wall. In this regard, patients with chronic inflammatory disease such as RA and SLE are at increased risk, which was attributed to the high levels of inflammatory mediators. However, less is known about the association of low-grade systemic inflammation with the cardiovascular risk.

Objectives: The aim of the present study was therefore to evaluate biomarkers representing low-grade systemic inflammation and their association with mortality in a large cohort of patients.

Methods: The Ludwigshafen Risk and Cardiovascular Health (LURIC) study included 3316 consecutive patients undergoing coronary angiography between June 1997 and May 2001 with a median follow-up of 9.9 years. Before coronary angiography fasting blood samples were collected and plasma levels of interleukin-6 (IL-6), C-reactive protein (CRP) and serum amyloid A (SAA) were measured by immunonephelometry. IL-6 and SAA polymorphisms were genotyped.

Results: During a median observation time of 9.9 years 949 deaths $(30.3 \%)$ occurred, of these 597 (19.2\%) died from cardiovascular events. Plasma levels of IL-6, CRP and SAA were associated with unstable CAD as well as established risk factors including type 2 diabetes, smoking, lower eGFR, lower triglycerides and lower HDL-C. In addition, IL-6 correlated with age, use of lipid lowering therapy and hypertension, SAA correlated with female gender and hypertension, and CRP correlated with female gender, lipid-lowering therapy and BMI. After adjustment for established cardiovascular risk markers and the other two inflammatory markers, SAA was found an independent risk factor for cardiovascular mortality after a short-term follow-up (1 year) with a HR of 1.41 (1.03 1.93, $\mathrm{p}=0.030)$, whereas IL-6 was identified as an independent risk factor for long-term follow-up (3, 5-9.9 years) with HRs of 1.21 (1.02-1.43, $\mathrm{p}=0.006), 1.22(1.06-1.40, \mathrm{p}=0.006)$ and 1.18 (1.07-1.31, $\mathrm{p}=0.001)$, respectively. Although 6 SNPs in the SAA gene were significantly associated with SAA plasma concentrations (none of the IL-6 SNPs associated with IL-6 levels), the genetic risk score was not associated with mortality. Conclusion: In our large, long-term LURIC cohort study we demonstrate for the first time prospectively that plasma levels of IL-6 and SAA are not only associated with cardiovascular risk factors and the prevalence of CAD but also independently predicted cardiovascular mortality. These findings underline the importance of low-grade systemic inflammation for the cardiovascular risk and the prognostic relevance of inflammatory biomarkers, independently predicting cardiovascular mortality. Although it was not the focus of this study, our findings might suggest that even lowgrade inflammation is unfavourable in chronic rheumatic disease.

Disclosure of Interests: None declared

DOI: 10.1136/annrheumdis-2019-eular.7919

\section{FRI0661 ASSOCIATIONS OF CURRENT AND CHILDHOOD SOCIOECONOMIC STATUS WITH PATIENT-REPORTED HEALTH OUTCOMES AMONG PATIENTS WITH KNEE OR HIP OSTEOARTHRITIS IN A FAMILY PRACTICE SETTING IN MEXICO CITY}

Julio Pisanty Alatorre ${ }^{1}$, Maria Victoria Goycochea Robles ${ }^{2} .{ }^{1}$ Instituto Mexicano del Seguro Social, HGZMF 8, Mexico, Mexico; ${ }^{2}$ Instituto Mexicano del Seguro Social, Unidad de Investigación en Epidemiología Clínica HGR1, Mexico, Mexico

Background: The life-course approach is a useful framework to understanding socioeconomic inequalities in the prevalence and outcomes of chronic disease ${ }^{1}$ and in particular in osteoarthritis (OA). ${ }^{2}$ Previous studies have measured Socioeconomic Status (SES) in 2 points of the life-course (childhood and current) and found consistent relationships between them and both prevalence and presence of worse outcomes, ${ }^{2-4}$ but this relationship has been little explored in the Latin-American region where exposure to low SES throughout the life-course is higher than in developed countries.

Objectives: Our aim was to investigate the association of current and childhood SES with functional status, quality of life and disability among patients with knee or hip OA.

Methods: We recruited patients with self-reported doctor-diagnosed knee or hip $O A$ in a social security family practice setting, excluding patients with other rheumatic diseases liable to causing secondary OA. We measured current SES by education, occupation type and estimated income using a validated tool ${ }^{5}$ and childhood SES by parental education and occupation type. We measured functional status, quality of life and disability using Spanish-language versions of the WOMAC, ${ }^{6} \mathrm{AMICAL}^{7}$ and HAQ-DI ${ }^{8}$ tools. We used Spearman correlations, ANOVA and multiple linear regression to test for significance, with a $p<0.05$ cutpoint.

Results: We recruited 146 patients with a mean age of 69.4 yr. $80 \%$ were female; $92 \%, 30 \%$ and $45 \%$ suffered from knee, hip and other join OA, respectively, while $60 \%$ had incomes in the upper 2 quintiles of the general Mexican population. We found current income to be the best pre dictor of WOMAC (Spearman's $\rho=-0.354, \quad \mathrm{p}<0.01)$, AMICAL $(\rho=-0.362$, $\mathrm{p}<0.01)$ and $\mathrm{HAQ}-\mathrm{DI}$ scores $(\rho=-0.335, \mathrm{p}<0.01)$, and education to be correlated with all three scores as well. Occupation type was significantly associated with AMICAL and HAQ-DI but not WOMAC scores. Paternal and maternal education, and maternal occupation type, were significantly associated only with AMICAL scores.

In multiple linear regression models were income and maternal education were entered separately, after adjusting for age, sex, BMI and presence of Diabetes and Hypertension, both variables were significantly associated with WOMAC, AMICAL and HAQ-DI scores (adj $\mathrm{r}^{2} 0.076-0.157, \mathrm{p}<0.05$ ). When both variables were entered into multiple linear regression models (adj $r^{2} 0.112$ and 0.110 ), significant associations remained between current income level and WOMAC and AMICAL scores $(p<0.01)$, as well as between maternal education and AMICAL scores $(p<0.05)$. Associations between both variables and HAQ-DI scores did not reach statistical significance $(p=0.07)$. 